\title{
Abscisic acid does not influence the subcellular distribution of the HYL1 protein from Arabidopsis thaliana ${ }^{\star}$
}

\author{
Joanna Lesicka-Górecka1 , Bogna Szarzyńska1 , Marta Sawczak ${ }^{1}$ Ivona Bagdiul ${ }^{1,2}$, \\ Paweł Górski ${ }^{1}$, Artur Jarmołowski ${ }^{1}$ and Zofia Szweykowska-Kulińska ${ }^{1 凶}$ \\ ${ }^{1}$ Department of Gene Expression, Institute of Molecular Biology and Biotechnology, Faculty of Biology; \\ ${ }^{2}$ Departament of Crystallography, Faculty of Chemistry, Adam Mickiewicz University, Poznan, Poland
}

Received: 27 April, 2008; revised: 03 July, 2008; accepted: 08 August, 2008

available on-line: 20 August, 2008

\begin{abstract}
HYL1 is a nuclear protein involved in the processing of miRNAs but its exact function remains unknown. Arabidopsis thaliana hyl1 mutants exhibit hypersensitivity to ABA. We decided to answer the question whether ABA affects the HYL1 protein localization within the cell and show that it does not. We also studied the expression of HYL1 in different tissues and organs. In this paper we show for the first time the expression profile of the HYL1 protein using anti-HYL1 antibodies. The protein is present in seedlings and mature plants in all organs studied, with the highest amount in inflorescences. A. thaliana HYL1 protein has several repetitions of a 28-amino-acid sequence at the C-terminus that confer protein instability. Our bioinformatic analysis of HYL1 homologs in different Brassica species shows that this repetition is typical only for Arabidopsis. This may suggest a relatively late evolutionary acquisition of the C-terminal domain.
\end{abstract}

Keywords: Arabidopsis thaliana, HYL1 protein, abscisic acid, subcellular distribution, expression profile

\section{INTRODUCTION}

The Arabidopsis HYL1 gene encodes a nuclear double-stranded RNA-binding protein involved in microRNA (miRNA) biogenesis. The hyl1 null mutant, designated hyponastic leaves 1, exhibits severe developmental defects, altered hormone response and reduced miRNAs level (Lu \& Fedoroff, 2000; Han et al., 2004; Vazquez et al., 2004). The HYL1 protein is a part of a large $(300-500 \mathrm{kDa})$, rather unstable complex (Song et al., 2007). It is suggested that DCL1 is also a component of the HYL1 complex and the interaction of HYL1 with DCL1 is important for the efficient and precise processing of at least some miRNA primary transcripts during plant miRNA biogenesis (Kurihara et al., 2006). Recent studies have shown that another protein, SERRATE (SE), is also involved in the accumulation of miRNA and contributes to normal plant development along with DCL1 and HYL1 (Yang et al., 2006). Results of bimolecular fluorescence complementation (BiFC) and fluorescence resonance energy transfer (FRET) experiments show that HYL1, DCL1 and SE interact in bodies located in the nucleus. It is likely that the DCL1-HYL1-SE nuclear bodies are involved in miRNA production since miRNA precursors are localized within these structures. Two protein markers of Cajal bodies, SmD3 and SmB, co-localize with HYL1, DCL1 and SE, but the DCL1-HYL1-SE complexes do not localize in the bodies identified by another marker of Cajal bodies - coilin (Fang \& Spector, 2007; Fujioka et al., 2007; Song et al., 2007). It has

${ }^{\square}$ Corresponding author: Zofia Szweykowska-Kulińska, Department of Gene Expression, Adam Mickiewicz University, Międzychodzka 5, 60-371 Poznań, Poland; phone and fax: (48) 61829 2730; e-mail: zofszwey@amu.edu.pl

"Presented at the XXXV Winter School "The Structure and Function of Protein and Nucleic Acids" organized by Faculty of Biochemistry, Biophysics and Biotechnology, Jagiellonian University, 23-27 February, 2008, Zakopane, Poland.

Abbreviations: ABA, abscisic acid; DCL1, dicer like 1; DRB, dsRNA-binding; dsRBM, dsRNA-binding motif; dsRBD, dsRNA-binding domain; dsRNA, double-stranded RNA; GFP, green fluorescent protein; miRNA, microRNA; HYL1, hyponastic leaves 1; NLS, nuclear localization signal; SE, serrate. 
already been suggested that SmD3-containing Cajal bodies take part in the processing of transcripts during formation of the A. thaliana RISC complex (Li et al., 2006).

It has been shown that at least several microRNAs play a role in the plant response to abiotic and biotic stresses (Sunkar et al., 2007). Tolerance to specific stresses is mediated mainly by the plant hormone abscisic acid (ABA) (Xiong et al., 2002). The A. thaliana hyl1 mutant exhibits hypersensitivity to ABA. Since it also shows impairment of the microRNA biogenesis, we decided to look for the possibility of an involvement of ABA in HYL1 subcellular localization. In this paper we show that ABA has no influence on the HYL1 localization in the cell. Western blot analysis of the HYL1 expression profile has revealed the presence of this protein in all plant organs with the highest amount present in the inflorescence, in agreement with earlier HYL1 mRNA expression data (Han et al., 2004).

\section{MATERIALS AND METHODS:}

Plants. All experiments were performed on Arabidopsis thaliana ecotype Columbia-0, growing at $22^{\circ} \mathrm{C}$ at 12 -h light, 12 -h dark photoperiod. Nicotiana tabacum var. xanthi plants were grown at $22^{\circ} \mathrm{C}$ at 16 $\mathrm{h}$ light, 8-h dark photoperiod.

Reagents. Anti-HYL1 rabbit polyclonal antibody was raised (Agrisera, Sweden) against the 15 aa C-terminal peptide (N-CMSGLKEAAFGSVET-C) predicted to be highly specific to HYL1 as described in (Han et al., 2004). Starlight ${ }^{\mathrm{TM}}$ Chemiluminescent Substrate and luminescence enhancer Opti-Membrane ${ }^{\mathrm{TM}}$ Reagent were from MP Biomedicals Inc. (Ohio, USA). TRIzol Reagent used for RNA isolation, as well as all materials for insect cell growth and transformation were from Invitrogen Life Technologies. The Talon affinity matrix for purification of $\mathrm{His}_{6}$-tagged proteins was from Clontech BD Biosciences. PVDF membranes were from Millipore or Amersham Biosciences. pGEM-T Easy and reverse transcriptase were purchased from Promega. Cellulase and macerozyme R10 were from Serva; drisellase and abscisic acid were from Sigma Aldrich. If not stated otherwise, enzymes were from Fermentas.

cDNA isolation from A. thaliana. Total RNA was extracted from mature leaves of hyl1 mutants and wild type plants using TRIzol Reagent, and the first strand of cDNA was synthesized using MMLV reverse transcriptase according to the manufacturer's protocol. The sequences of HYL1 cDNA - specific primers were as follows: 5'-CTGGTCGACAATGACCTCCACTGATGTTTC-3' (forward) and 5'-GGATCCTGCGTGGCTTGCTTCTGTC-3' (reverse). After amplification the cDNA was cloned into the pGEM-T Easy vector.
Western blot analysis. Total protein extract was obtained from mature leaves of $A$. thaliana wild type plants and homozygous hyl1 T-DNA insertion mutants (SALK_064863). For Western blotting analysis, $10 \mu \mathrm{g}$ of total protein extracts was loaded on a $10 \%$ polyacrylamide gel. For HYL1 expression profile analysis, tissues were collected from 6-dayold seedlings, 32-day-old roots, 32-day-old rosette leaves, 37-day-old stem leaves and inflorescences. The proteins were subjected to electrophoresis and electrotransferred to PVDF Immobilon-P membrane. The membranes were blocked overnight at $4^{\circ} \mathrm{C}$ with $3 \%$ BSA (bovine serum albumin) solution in TBS-T (20 mM Tris/ $\mathrm{HCl}, 137 \mathrm{mM} \mathrm{NaCl}, 0.1 \%$ Tween 20) buffer, and subsequently incubated with anti-HYL1 antibodies and with anti-rabbit IgG antibodies conjugated with alkaline phosphatase. The targeted protein bands were visualized by chemiluminescence using Starlight ${ }^{\mathrm{TM}}$ Chemiluminescent Substrate and luminescence enhancer Opti-Membrane ${ }^{\mathrm{TM}}$ Reagent.

HYL1 immunoprecipitation from A. thaliana protein extract. In a standard immunoprecipitation reaction, $0.5 \mathrm{~g}$ of Arabidopsis leaves was ground into fine powder under liquid nitrogen and then homogenized in $1 \mathrm{ml}$ of extraction buffer $\mathrm{pH} 7.5(50 \mathrm{mM}$ Tris/ $\mathrm{HCl}, 50 \mathrm{mM} \mathrm{NaCl}, 0.1 \%$ Triton-X ) containing protease inhibitor cocktail (1 tablet/10 ml; Roche). Cell debris was removed by centrifugation at $4^{\circ} \mathrm{C}$ $(22000 \times g, 20 \mathrm{~min})$. The supernatant was collected and protein concentration was determined using BioRad Protein Assay (Bio-Rad). Protein concentrations were adjusted to $10 \mathrm{mg} / \mathrm{ml}$ with extraction buffer and $0.5 \mathrm{ml}$ of extract was precleared by incubation with $50 \mu \mathrm{l}$ of Protein A Sepharose (Amerham Biosciences) at $4^{\circ} \mathrm{C}$ for $30 \mathrm{~min}$. Precleared extracts were then incubated with $20 \mu \mathrm{l}$ of anti-HYL1 antibodies and $30 \mu \mathrm{l}$ of Protein A Sepharose at $4^{\circ} \mathrm{C}$ for $2 \mathrm{~h}$. Immunoprecipitates were washed three times $(20 \mathrm{~min}$. each) in a washing buffer $\mathrm{pH} 7.5(50 \mathrm{mM}$ Tris/ $\mathrm{HCl}$, $150 \mathrm{mM} \mathrm{NaCl}, 0.1 \%$ Triton-X).

Protoplast transfection and localization studies. The cloned HYL1 cDNA was inserted into the pDEDH72-eGFP vector (a kind gift from W. Filipowicz) at the N-terminal end of the GFP-coding sequence by using the SalI and BamHI sites. The HYL1-GFP chimeric gene was expressed from the cauliflower mosaic virus $35 \mathrm{~S}$ promoter. Isolation, purification and transient transfection of A. thaliana and N. tabacum protoplasts were carried out as described by (Prols et al., 1988; Li et al., 2002), respectively.

Protoplasts were transfected with recombined pDEDH72-HYL1-eGFP plasmid. PEG-mediated transfection was applied to introduce plasmids into the protoplasts of $A$. thaliana and N. tabacum var. xanti. Thirty micrograms of plasmid DNA was used for single transfection. After $24 \mathrm{~h}$ of incubation at room temperature, transient expression of HYL1-GFP was 
observed in a confocal Zeiss LSM5 10 microscope using a plan-apochromat $63 \times / 1.4$ Oil DIC objective. The argon laser $477 \mathrm{~nm}$ line and the helium-neon laser $543 \mathrm{~nm}$ line were used for the fluorescence excitation and detection, respectively. In the case of the influence of $\mathrm{ABA}$ on the subcellular localization of HYL1, $50 \mu \mathrm{M}$ ABA solution was prepared in 0.02 $\mathrm{M} \mathrm{NaOH}$ and added to the protoplast suspension. Subsequently, protoplasts were incubated for 20, 45 or $180 \mathrm{~min}$ and the localization of the fused protein was observed.

Expression of HYL1 protein in baculovirus system. Recombinant protein isolation and purification. The HYL1 cDNA was cloned from the pGEM-T Easy vector into the BamHI and EcoRI sites of the pFAST-Bac HT B vector (Invitrogen). All procedures were conducted as described in the Invitrogen Life Technologies Instruction Manual. The yield of the HYL1 protein in the baculovirus Bac-to-Bac system was $1.5-2 \mathrm{mg} /$ liter of medium.

Protein disorder prediction. Protein disorder was predicted using the VL-XT Predictor (Romero et al., 1997; 2001; Li et al., 1999) at PONDR (Predictor of Natural Disordered Regions; www.pondr.com) server. Domains in HYL1 were assigned according to (St Johnston et al., 1992).

Homolog searches and analysis. In order to find homologs of the HYL1 protein (NP_563950.1), the pBLAST algorithm (Altschul et al., 1997; Schäffer et al., 2001) was used to search the non-redundant version of the current NCBI sequence databases. All homologous sequences from the Brassicaceae family were aligned using the T-coffee algorithm (Notredame et al., 2000). An illustration of the alignment was prepared with the Jalview program (Clamp et al., 2004).

\section{RESULTS}

\section{HYL1 expression profile}

To study the HYL1 expression profile at the protein level we decided to produce antibodies against HYL1 using a protein peptide fragment (401-415 aa), according to the procedure described by (Han et al., 2004). In our Western blot experiments, the rabbit antibodies raised against the HYL1 short fragment recognized specifically the HYL1 protein and another, unknown protein from wild type plants. The same analysis with a protein extract prepared from the T-DNA insertion mutant of the HYL1 gene showed no signal at the level of the HYL1 protein, however, the unknown protein was still detected (Fig. 1A).

To confirm the presence of the HYL1 protein during different stages of plant development and in different organs, we isolated total proteins from
A. thaliana seedlings, roots, rosette, and stem leaves and inflorescences and subjected then to Western blotting. The HYL1 protein was present in young developing plants and in all organs of the mature ones, with the highest HYL1 expression level in the inflorescence (Fig. 1B).

To check whether the anti-HYL1 antibodies recognize the native structure of HYL1 we immunoprecipitated the HYL1 protein from protein extracts prepared from Arabidopsis wild type plants and from the hyl1 mutant. Western blot analysis revealed the presence of the HYL1 protein on the blot containing precipitates from the wild type plant and its absence in the case of the hyll mutant. This suggests that the HYL1 antibodies recognize the native form of the HYL1 protein and can be used for identification of other proteins in the HYL1 complex by coimmunoprecipitation (Fig. 1C). Immunoprecipitation of the HYL1 protein from the wild type and the hyl1 mutant protein extracts did not give any signal in Western blot at the gel migration position of HYL1 in the control experiment when pre-immune rabbit serum was used (not shown).

The influence of abscisic acid on the subcellular localization of the HYL1 protein

The A. thaliana hyl1 mutant shows hypersensitivity to abscisic acid resulting in a complete inhibition of seed germination in the presence of $0.4 \mu \mathrm{M}$

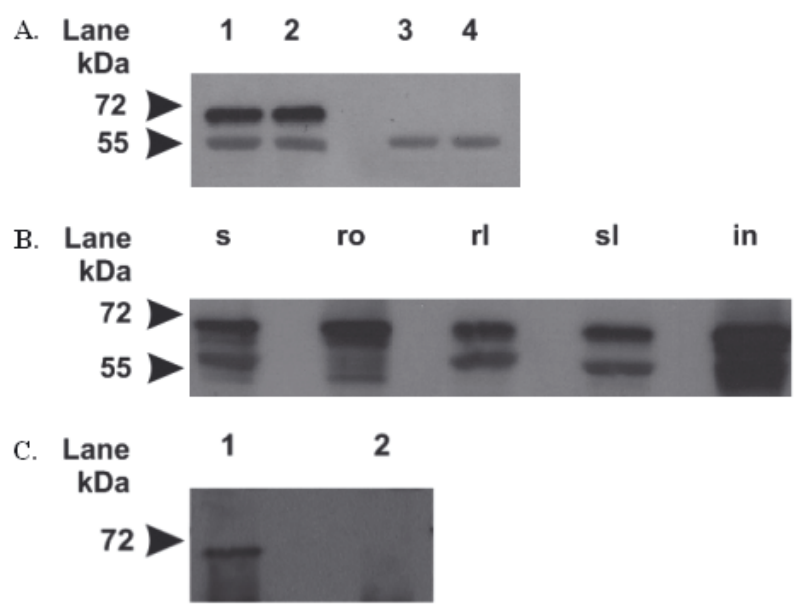

Figure 1. Western blot detection of HYL1 expression with anti-HYL1 antibody.

A. The HYL1 protein expressed in Arabidopsis thaliana; lanes 1 and 2, total protein isolated from rosette leaves of wild type plants, lanes 3 and 4 , total protein isolated from rosette leaves of hyl1 T-DNA insertion mutants. B. Total protein isolated from seedlings (s), roots (ro), rosette leaves ( $\mathrm{rl}$ ), stem leaves (sl) and inflorescences (in) of wild type plants. C. Immunoprecipitation of the HYL1 protein expressed in $A$. thaliana with anti-HYL1 antibody; lane 1, HYL1 protein immunoprecipitated from rosette leaves of wild type plants; Lane 2, HYL1 protein immunoprecipitated from rosette leaves of hyl1 T-DNA insertion mutants. 

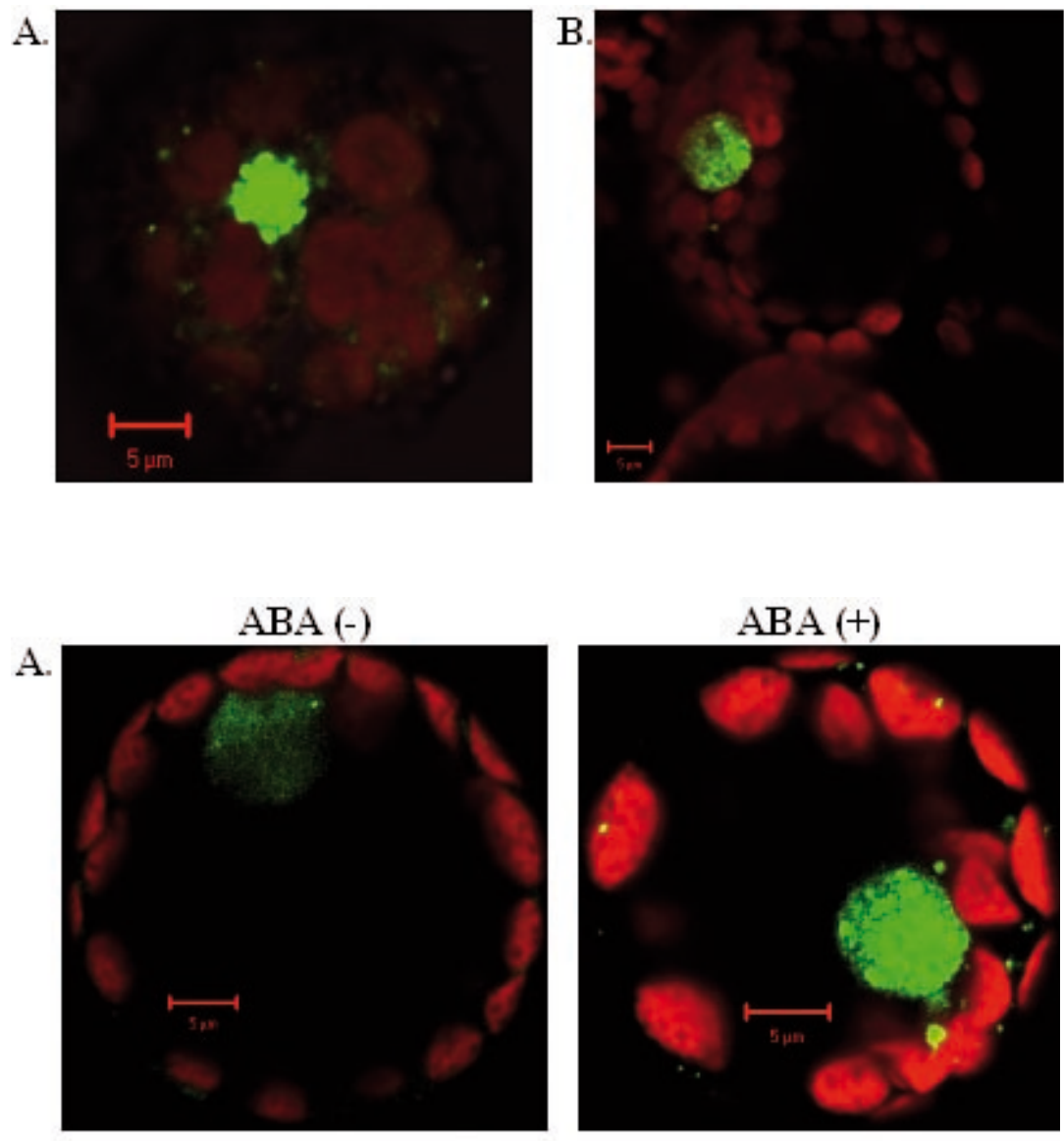

B.
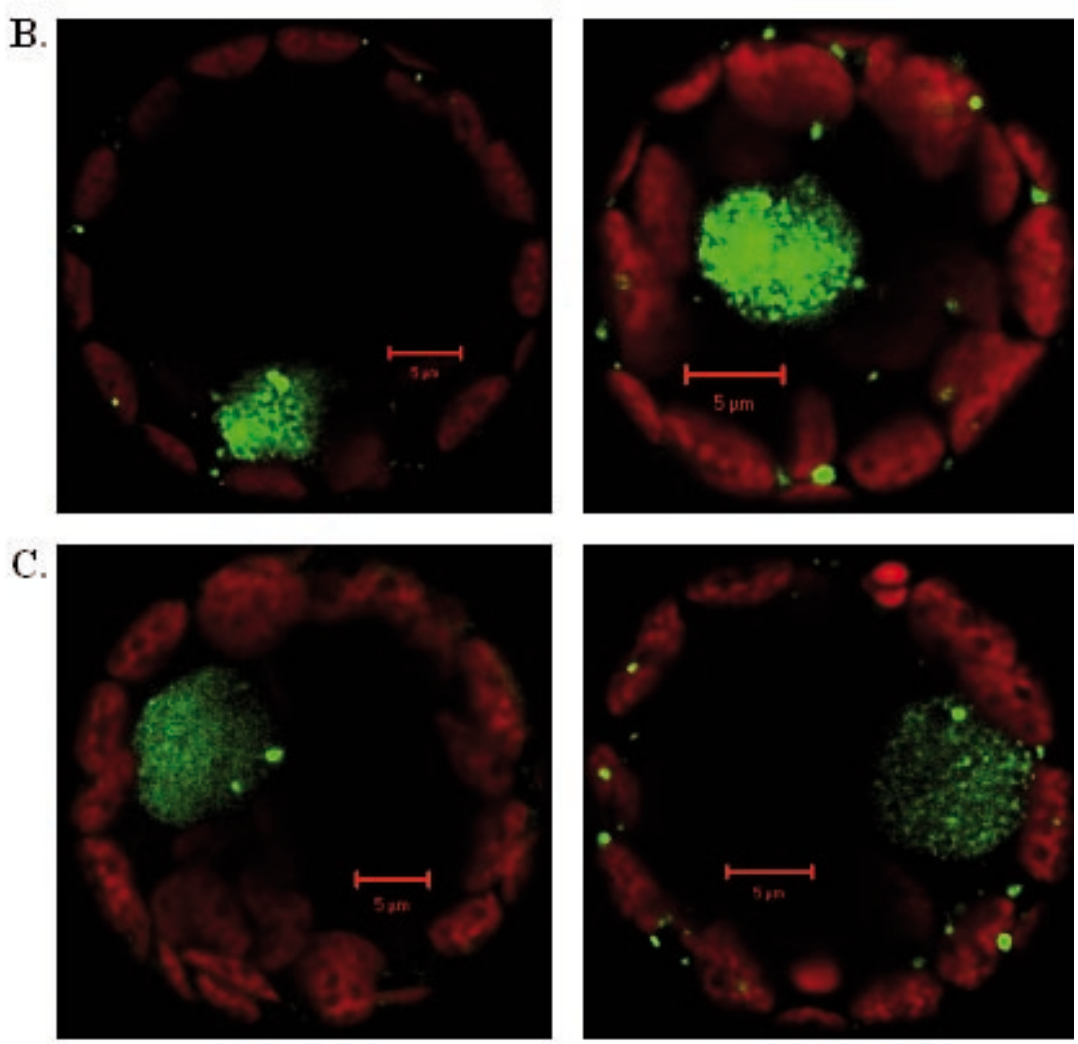

Figure 3. HYL1-GFP localization in tobacco protoplasts in the presence or absence of ABA.

(A), (B), and (C): transfected protoplasts after 20, 45, and $180 \mathrm{~min}$ of treatment with buffer (images on the left) or $50 \mu \mathrm{M}$ ABA (images on the right).
Figure 2. Transient expression of the HYL1-GFP fusion protein (green) in A. thaliana (A) and N. tabacum var. xanthi (B) mesophyll protoplasts. Red fluorescence comes from chloroplasts. Because of strong chlorophyll autofluorescence, $477 \mathrm{~nm}$ line was used for excitation instead of $488 \mathrm{~nm}$ standard one. Further details are presented in Materials and Methods.

ABA (not shown; Lu \& Fedoroff, 2000). To answer how ABA is involved in the HYL1 action we decided to study the influence of this hormone on the subcellular localization of the HYL1 protein. First we performed transfections with a HYL1-GFP construct into A. thaliana and N. tabacum protoplasts. As expected, in A. thaliana the fusion protein was present in the nucleus and formed small, well-defined speckles. We obtained the same results when tobacco protoplasts were used (Fig. 2). Presumably as a result of the very strong overexpression of the fusion protein, some small green speckles were also present outside the nucleus. This experiment proved that both systems tested can be used to study the subcellular localization of the A. thaliana HYL1 protein. Since tobacco protoplasts are larger and easier to isolate and transfect, we decided to use them in further experiments. The protoplasts were transfected with the HYL1-GFP construct and incubated in darkness for $24 \mathrm{~h}$. The protoplasts were divided into two groups: one was incubated in a medium containing $50 \mu \mathrm{M}$ $\mathrm{ABA}$, the second in a medium without the hormone. HYL1-GFP localization was observed in the protoplasts from both groups after 20,45 or $180 \mathrm{~min}$ from $\mathrm{ABA}$ application. In all the cases we observed the same HYL1-GFP localization within the cell, namely, in multiple, well-defined bright 


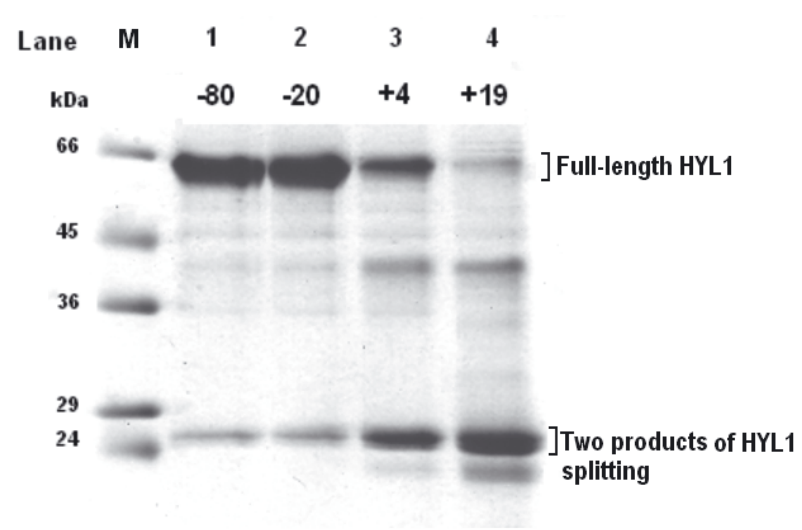

Figure 4. Stability of full-length HYL1 protein stored at different conditions for three weeks.

Polyacrylamide denaturing gel (15\%) stained with Coomassie Brilliant Blue. Lanes: $\mathrm{M}$, molecular mass marker; 1 , protein sample stored at $-80^{\circ} \mathrm{C}$ (control); 2 , at $-20^{\circ} \mathrm{C}$; 3 , at $+4^{\circ} \mathrm{C}$; 4 ; at $+19^{\circ} \mathrm{C}$ in the following buffer: $20 \mathrm{mM}$ Tris/ $\mathrm{Cl}, \mathrm{pH} 8.8,200 \mathrm{mM} \mathrm{NaCl}$ and $250 \mathrm{mM}$ imidazole.

spots in the nucleus (Fig. 3). Thus, ABA does not influence the subcellular distribution of the HYL1 protein.

\section{HYL1 overexpression and stability}

The efficiency of HYL1 overexpression in the baculovirus system was high: $1.5-2.0 \mathrm{mg} /$ medium liter. Since the expression efficiency was good, we intended to use the protein for crystallization studies. However, we found that the protein is unstable. After three weeks of storage at $+4^{\circ} \mathrm{C}$ about $50 \%$ of the protein was degraded, while storage at $+19^{\circ} \mathrm{C}$

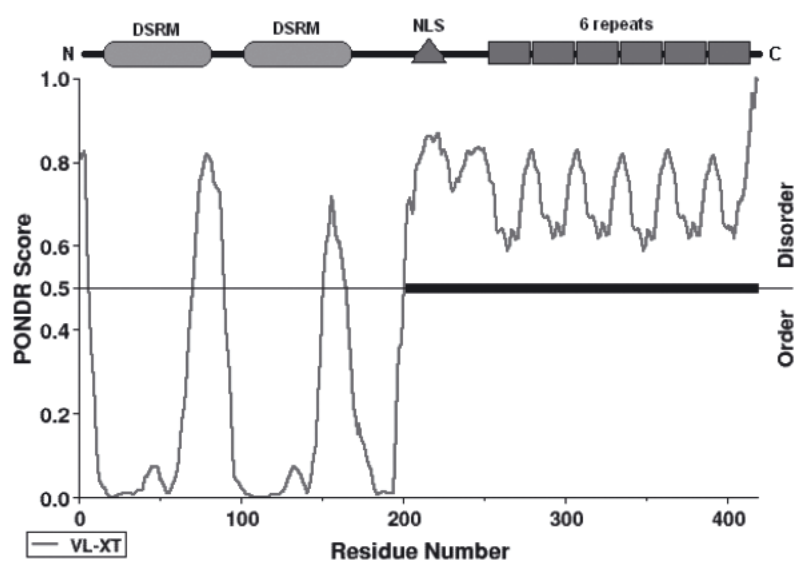

Figure 5. Analysis of the HYL1 structure (top) with PONDR prediction of ordered/disordered regions program (bottom).

Top: DSRM, putative dsRNA-binding motifs; NLS, putative bipartite nuclear localization sequence. Bottom: Each residue ( $X$-axis) is assigned a disorder score $(Y$-axis) by the predictor based on the attributes of amino acids surrounding the residue. Predicted scores $\geq 0.5$ indicate disorder. resulted in about $90 \%$ degradation (Fig. 4). The two major degradation products have almost the same molecular masses (about $24 \mathrm{kDa}$, Fig. 4) and are present in similar amounts. This would mean that the protein is likely to break down spontaneously into two parts: one containing the dsRBD domain and the other one containing the NLS signal and six 28-amino-acid repeats (as deduced from the HYL1 protein primary structure, see Fig. 5).

The HYL1 sequence was analyzed with PONDR to predict the ordered/disordered regions. The results show that the C-terminal part containing NLS and the 28-amino-acid repeats is intrinsically disordered (ID, unstructured), while the N-terminal part of the protein exhibits a highly ordered structure (Fig. 5). It is clearly visible that a dramatic change in the HYL1 structure takes place in the middle of the protein, and it can also explain the observed protein degradation into two parts.

\section{DISCUSSION}

The existence of microRNAs have been discovered as a consequence of intense work on the identification of short RNAs involved in RNA interference process (Szweykowska-Kulińska et al., 2003; Lesicka et al., 2004). HYL1 protein is involved in the maturation of pre-miRNA precursors. In this paper we have shown for the first time the expression profile of the HYL1 protein using anti-HYL1 antibodies. The protein is present in seedlings and mature plants in all the studied organs. However, it predominates in the inflorescence, which is in concordance with the mRNA expression profile data presented in the Arabidopsis eFP Browser (http://www.bar.utoronto. ca/efp/cgi-bin/efpWeb.cgi). This may be explained by the involvement of many HYL1-dependent microRNAs in the generative processes.

We also present data concerning the subcellular localization of HYL1 in the presence or absence of ABA. It has been found that the RNA-binding protein AKIP1 from Vicia faba (interacting with the abscisic-acid-activated protein kinase AAPK), which is usually dispersed in the guard cell nuclei, redistributes and concentrates in subnuclear foci after ABA treatment (Li et al., 2002). The same has been reported for the RNA-binding UBA2a protein from $A$. thaliana, the closest homologue of the $V$. faba AKIP1 protein. It also reorganizes in the nucleus in "speckles" in response to ABA and is possibly involved in the regulation of RNA metabolism during ABA signaling (Riera et al., 2006). Since HYL1 is an RNA-binding protein and the hyl1 mutant exhibits altered $\mathrm{ABA}$ response we hypothesized that $\mathrm{ABA}$ may change HYL1 subcellular localization. HYL1 has earlier been reported to be present in the nucle- 
us in both dispersed and concentrated forms in subnuclear foci (Lu \& Fedoroff, 2000). Our results show that $\mathrm{ABA}$ does not substantially influence the distribution of the HYL1-GFP protein in the cell. However, more concentrated HYL1-containing foci within the nucleus are observed in the case of ABA-treated protoplast (see Fig. 2). High-resolution images have to be obtained for an unambiguous statement about the possible changes in the subnuclear localization of HYL1-containig bodies.

Two hypotheses explaining how ABA is involved in the HYL1 action are possible: (i) HYL1 itself is a negative regulator of a putative intermediate that acts in the ABA signaling pathway or (ii) microRNA(s) that requires HYL1 for efficient maturation controls the level of the putative negative regulator in ABA signal transduction. In both cases an altered response to ABA will lead to hypersensitivity to this hormone, as it is observed in the hyl1 mutant. We think the second hypothesis is more likely and we are preparing experiments to find all targets of microRNAs that require HYL1 protein for their biogenesis. Other $A$. thaliana mutants have been described that show hypersensitivity to $\mathrm{ABA}$ and in which the RNA metabolism is altered. These are: abh1, cbp20, sad1, and sad2. The ABH1 and CBP2O genes encode two cap-binding proteins - CBP80 and CBP20, respectively, and are involved in premRNA splicing (Hugouvieux et al., 2001). The SAD1 protein belongs to Sm-like snRNP proteins required for mRNA splicing, export, and degradation (Xiong et al., 2001). Localization of the SAD1 protein was studied in Arabidopsis. It was found in the nucleus and cytoplasm (Kufel J., Kruszka K., unpublished). The SAD2 protein, also mainly localized in the nucleus, belongs to the $\beta$-importin family and is involved in nuclear transport (Verslues et al., 2006). These observations suggest a role for nuclear proteins and nuclear transport in ABA signal transduction. However, there are no reports indicating redistribution of these proteins within the cell/nucleus when ABA is present. Thus the AKIP1/UBA2a proteins remain to be the only two proteins for which ABA-dependent localization has been observed.

The question about the exact function of the HYL1 protein remains unanswered. Analysis of the hyl1 mutant showed that the protein is involved in the processing of several miRNAs, probably during the interactions with the main miRNA, processing enzyme in plants, DCL1 (Hiraguri et al., 2005). We have successfully overexpressed the HYL1 protein from A. thaliana in baculoviruses, however, our attempts to crystallize it have been so far unsuccessful since the protein spontaneously splits into two parts. The HYL1 protein belongs to the DRB protein family (dsRNA-binding) and has two dsRNA-binding domains (dsRBD1 and dsRBD2) in its N-terminal half, a bipartite nuclear localization motif and six repetitions of a 28-amino-acid sequence at the C-terminus. Only the dsRBD1 domain of HYL1 is essential for dsRNA binding in vitro, whereas dsRBD2 contributes to its protein-protein interactions (Hiraguri et al., 2005). It is presumed that the C-terminal repeats are also responsible for binding other unknown protein partners (Lu \& Fedoroff, 2000). To answer the question why the HYL1 protein breaks up so rapidly, we used the PONDR program for protein disorder prediction. The results indicate that the protein could be divided into two parts: a highly structured N-terminal part containing the two dsRBDs domains and a highly unstructured C-terminal part containing the NLS signal and the six 28-amino-acid repeats. This could be the reason why the disordered C-terminus destabilizes the protein, resulting in its fragmentation into two parts (Dobson, 1999). The experiments were done in vitro using purified HYL1 protein. In vivo HYL1 may be stable since it functions in a complex of about 300-500 kDa (Han et al., 2004; Song et al., 2007) with at least one known protein (DCL1), which might stabilize the C-terminus and prevent HYL1 from degradation detected in vitro. What is the function of the HYL1 disordered part in addition to targeting the protein to the nucleus? It is known that intrinsically disordered proteins and regions may carry out numerous biological functions including cell signaling, molecular recognition, and protein modifications (including phosphorylation) (Iakoucheva et al., 2004; Radivojac et al., 2007). However, hyl1 plants transformed with a HYL1 cDNA fragment encoding only two N-terminal dsRBDs displayed a complete rescue of the phenotypes conferred by the hyl1 mutation ( $\mathrm{Wu}$ et al., 2007). Moreover, a comparison of these transgenic and wild type plants revealed that the dsRBDs of HYL1 restore the accumulation of miR165, miR160 and miR319, and guide the posttranscriptional silencing of the REV and ARF17 genes. These findings indicate that the dsRBDs are essential for HYL1 function and the C-terminal NLS and six repeats are not required ( $\mathrm{Wu}$ et al., 2007). This statement can be strengthened by our bioinformatic analysis of HYL1 homologs in different Brassica species: A. thaliana is the only species where the HYL1 protein has several repetitions of the 28-amino-acid sequence at the C-terminus. All other species only have a single sequence motif in this region (Fig. 6). Furthermore, even between $A$. thaliana ecotypes there are differences in the number of the repeated sequences. In the Wassilevskija, Landsberg erecta and Nossen ecotypes there are five repetitions of the 28-amino-acid motif, in contrast to the Columbia ecotype which has six (Han M.-H., personal communication). This observation may suggest a relatively late evolutionary acquisition of the C-terminal domain. The function of the HYL1 C-terminal part remains obscure. 
NP_563250.1 HYl [Avabidopsis thaliand] CAC05659.1 RBP2 [Brassios oleraces var. capizata] AAT77419.1 LH2-0 [BRssica napus]

AAT77416, LHI [Brassicd olevosed var, capzatd] AAT77417,1 LH2 [Prassicas oleraces rar. captato] CAB93934.1 BepLH [Brassioa rapa subsp. peldhensis] AAT77415, [Bassica rapa subsp, chinensis] CAD59426.1 [Brassicis overaces var. gongybodes] CAD59425.1 (Brassioa oleraeca var. batrytis] CADS9427.1 [Brassica oleracea var, itakiea] CADS9424.1 [Brsssiced oleraced var. acephulas] CAD59423.1 [Brassioa rapa subsp. rapa]

MP_56J850.1 HYL1 [Arabidopsis thalians] CAC05659.1 RBP2 [Brassica oloracea var. captata) AAT77419.1 LH2-0 [Basticd ndpus] AAT7746.1 LHI (Brassioa oleracea var. capitata) AAT77417.1 LHE [Brassica oloracea var. captata) CAB93934 1 BepLH [Bassicd rapd susps, pelonensis] AAT77415.1 [Brassica raps subsp. ohinensis] CADS9426. ' (Brassios oloraces var. gongyodos; CAD59425, [Bassicd olerasted var. Aotrytis] CAD59427.1 [Brassica olersces var. itsafica] CAD59424.1 [Brassios olevaces var. acephala] CAD59423 1 [Bassica rapal subsp, rapal)

MP_563050.1 HYL1 [Arabidopsis thaliana] CAC05659, 1 RBP2 [Brassica oleraced war. capirata] AAT77419.1 LH2-o [Brassica napus] AAT77416.1 LH' [Brassioa oloracea var. capitata) AAT77417,1 LH2 [Brassica oleracea var, eaphata] CA B\$O2034.1 BepLH (Brassicd rapd subsp, pelinensis) AMT77415.1 [Brassioa rapa subsp. ohinensis] CAD59426 1 [Bassica oleraced var, gongyodes] CA059425.5 [Brassicas oleraces var. botrytis] CAD59427.1 (Bassicas oleraces var. itafos) CAD59424. 1 [Brassica oleraced var, deephala] CAD59423, I [Erassica rapd subsp. rapa]

NP_563050.1 HYL1 Aabidopsis thallana) CAC05659.1 RBP2 [Bassica alepeed var. eapitata] AAT77419.1 LH20 [Brassica napus] AAT7746.1 LHI /Brassioa aleracea var. oapitata/ AAT77417,1 LH2 [Bassica aleracea var, capitata] CAE\$0934.1 BepLH [Brassica rapd subsp. peloinen. CADS9426.1 [Brassica olaracea var, gongyiodes] CADS9425.1 [Brassices oleraced var. Botritis] CAD59427.1 (Brassicas oleraoes var. italios) CADS9424.1 [Bassica oleracea var, acephala] CADS9423 1 [Basssicd and sidsp. rapd]

NP_56950.1 HYL1 [Arabidopsis thaliand] CAC05659.1 RBP2 (Brassica aleraced var. eapitata) AAT77419.1 LH2-0 (Brassica napus)

AAT77416.1 LHI /Brasticd alepced war. eapitata? AAT77417.1 LH2 [Bassica aleraces var. capitata] CABs3s34.1 BepLH [Brassica rapa subsp. poidinonsis) AAT77415.1 [Brassica rapa sudsp. ehinensis] CAD59426.1 [Brassics oleraces var. songylodes] CADS9425.1 (Brassica oleracea var. botrytis) CADS9427.1 [Brassica olevacea var, thakica] CAD59424.1 [Rrassics oleraces var. scephals] CADS9423.1 (Brassica raps subsp. rapa)

NP_563850.1 HYl [Aabidopsis thalland] CAC05659.1 RBP2 [Brassica oleraces var. captata] AAT77419.1 LH2-0 [Resssica napus]

AAT77416, LHI [Brassicd olecosed var, edpezata] AAT77417.1 LH2 [Brassica olersces var. capizata]

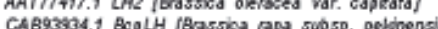
AAT77415.1 [Bassica rapa susp, chinensis] CADS9426.1 [Brassicice oleraced var. gongybodes] CAD59425.1 (Brassioa oleracea var. botrytis] CADS9427,1 [Brassica oleracea var, takica] CADS9424.1 [Brsssicted oteraced var. acephais] CAD59423.1 [Brassioa rapa subsp. rapa]

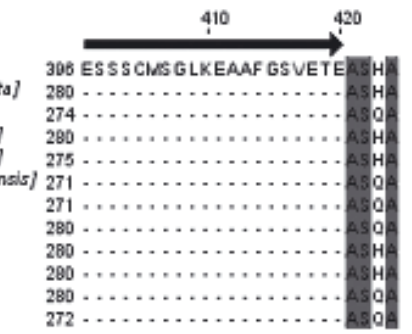
$410 \quad 420$

$\begin{array}{llll}30 & 40 & 50 & 60\end{array}$

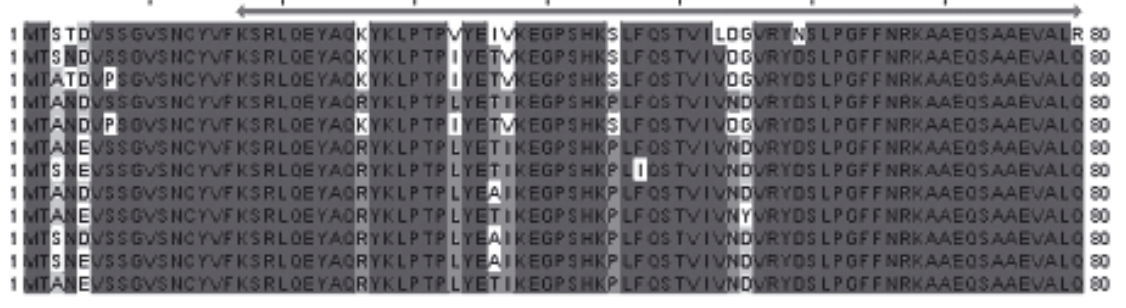

$900 \quad 100 \quad 110 \quad 120 \quad 130 \quad 150$

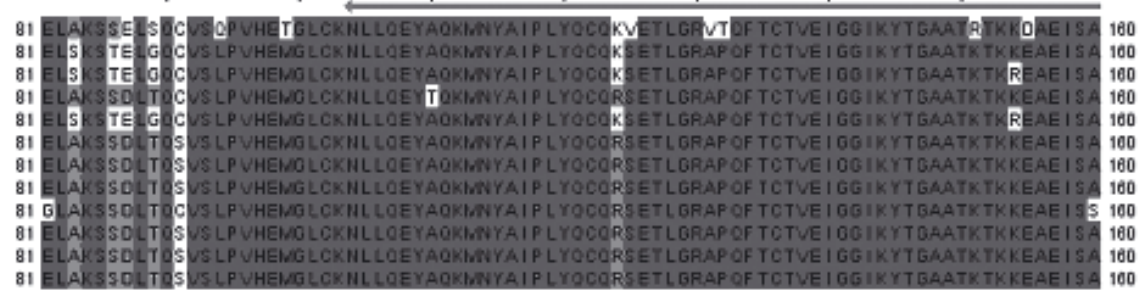

$170 \quad 100 \quad 190 \quad 200 \quad 210 \quad 220 \quad 230$
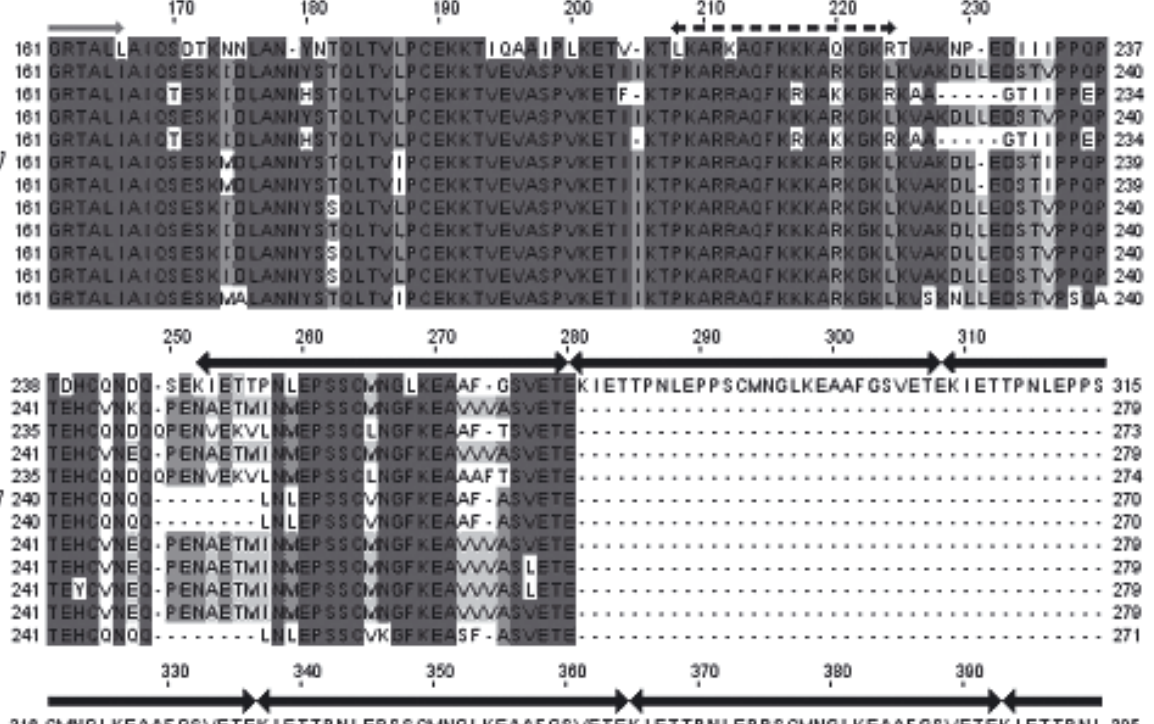

310 CMNOL KEAAF OSVETEKIETTPNLEP \$ \$CMNOL KEAAF OSVETEK IETTPNLEPP \$CMNOLKEAAF OSVETEKIETTPNL 395
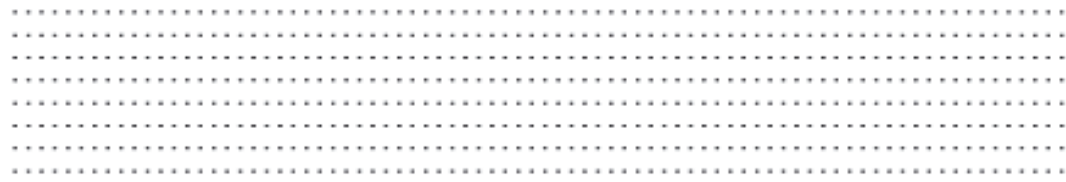
. .

Figure 6. Alignment of the amino-acid sequences of HYL1 homologs from A. thaliana and Brassica species. Black thick arrows above alignment indicate C-terminal repeats in HYL1 sequence.

\section{Acknowledgements}

We thank Professor Mariusz Jaskólski for many helpful suggestions on research and manuscript. We also want to thank Dr. Slawomir Samardakiewicz from the Laboratory of Electron and Confocal Microscopy, Adam Mickiewicz University, for his help during experiments done using confocal microscopy. 
This work was supported by the Ministry of Sciences and Higher Education grant: PBZ-KBN-110/ P04/2004.

\section{REFERENCES}

Altschul SF, Madden TL, Schäffer AA, Zhang J, Zhang Z, Miller W, Lipman DJ (1997) Gapped BLAST and PSIBLAST: a new generation of protein database search programs. Nucleic Acids Res 25: 3389-3402.

Clamp M, Cuff J, Searle SM, Barton GJ (2004) The Jalview Java Alignment Editor. Bioinformatics 20: 426-427.

Dobson CM (1999) Protein misfolding, evolution and disease. Trends Biochem Sci 24: 329-332.

Fujioka Y, Utsumi M, Ohba Y, Watanabe Y (2007) Location of a possible miRNA processing site in SmD3/SmB nuclear bodies in Arabidopsis. Plant Cell Physiol 48: 1243-1253.

Han M-H, Goud S, Song L, Fedoroff N (2004) The Arabidopsis double-stranded RNA-binding protein HYL1 plays a role in microRNA-mediated gene regulation. Proc Natl Acad Sci USA 101: 1093-1098.

Hiraguri A, Itoh R, Kondo N, Nomura Y, Aizawa D, Murai Y, Koiwa H, Seki M, Shinozaki K, Fukuhara T (2005) Specific interaction between Dicer-like proteins and HYL1/DRB-family dsRNA-binding proteins in Arabidopsis thaliana. Plant Mol Biol 57: 173-188.

Hugouvieux V, Kwak JM, Schroeder JI (2001) An mRNA cap binding protein, $\mathrm{ABH} 1$, modulates early abscisic acid signal transduction in Arabidopsis. Cell 106: 477487.

Iakoucheva LM, Radivojac P, Brown CJ, O'Connor TR, Sikes JG, Obradovic Z, Dunker AK (2004) The importance of intrinsic disorder for protein phosphorylation. Nucleic Acids Res 32: 1037-1049.

Kurihara Y, Takashi Y, Watanabe Y (2006) The interaction between DCL1 and HYL1 is important for efficient and precise processing of pri-miRNA in plant micro-RNA biogenesis. RNA 12: 206-212.

Lesicka J, Piontek P, Szweykowska-Kulinska Z, Jarmolowski A (2004) siRNAs and miRNAs: small RNA molecules for big tasks. Acta Phys Plant 26: 363-368.

Li CF, Pontes O, El-Shami M, Henderson IR, Bernatavichute YV, Chan SW, Lagrange T, Pikaard CS, Jacobsen SE (2006) An ARGONAUTE 4-containing nuclear processing center colocalized with cajal bodies in Arabidopsis thaliana. Cell 126: 93-106.

Li J, Kinoshita T, Pandey S, Ng CK, Gygi SP, Shimazaki K, Assmann SM (2002) Modulation of an RNA-binding protein by abscisic-acid-activated protein kinase. $\mathrm{Na}$ ture 418: 793-797.

Li X, Romero P, Rani M, Dunker AK, Obradovic Z (1999) Predicting protein disorder for $\mathrm{N}-, \mathrm{C}-$, and internal regions. Genome Informatics 10: 30-40.

Lu C, Fedoroff N (2000) A mutation in the Arabidopsis HYL1 gene encoding a dsRNA binding protein affects responses to abscisic acid, auxin and cytokinin. Plant Cell 12: 2351-2365.
Notredame C, Higgins D, Heringa J (2000) T-Coffee: A novel method for multiple sequence alignments. J Mol Biol 302: 205-217.

Prols M, Topfer R, Schell J, Steinbiss HH (1988) Transient gene expression in tobacco protoplasts. Plant Cell Rep 7: $221-224$

Radivojac P, Iakocheva LM, Oldfield CJ, Obradovic Z, Uversky VN, Dunker AK (2007) Intrinsic disorder and functional proteomics. Biophys J 92: 1439-1456.

Riera M, Redko Y, Leung J (2006) Arabidopsis RNA-binding protein UBA2a relocalizes into nuclear speckles in response to abscisic acid. FEBS Lett 580: 4160-4165.

Romero P, Obradovic Z, Dunker AK (1997) Sequence data analysis for long disordered regions prediction in the calcineurin family. Genome Informatics 8: 110-124.

Romero P, Obradovic Z, Li X, Garner E, Brown C, Dunker AK (2001) Sequence complexity of disordered protein. Proteins 42: 38-48.

Song L, Han M-H, Lesicka J, Fedoroff N (2007) Arabidopsis primary microRNA processing proteins HYL1 and DCL1 define a nuclear body distinct from the Cajal body. Proc Natl Acad Sci USA 13: 5437-5442.

Schäffer AA, Aravind L, Madden TL, Shavirin S, Spouge JL, Wolf YI, Koonin EV, Altschul SF (2001) Improving the accuracy of PSI-BLAST protein database searches with composition-based statistics and other refinements. Nucleic Acids Res 29: 2994-3005.

Sunkar R, Chinnusamy V, Zhu J, Zhu JK (2007) Small RNAs as big players in plant abiotic stress responses and nutrient deprivation. Trends Plant Sci 12: 301-309.

St Johnston D, Brown NH, Gall JG, Jantsch M (1992) A conserved double-stranded RNA-binding domain. Proc Natl Acad Sci USA 89: 10979-10983.

Szweykowska-Kulinska Z, Jarmolowski A, Figlerowicz M (2003) RNA interference and its role in the regulation of eucaryotic gene expression. Acta Biochim Polon 50: 217-229.

Vazquez F, Gasciolli V, Crete P, Vaucheret H (2004) The nuclear dsRNA binding protein HYL1 is required for microRNA accumulation and plant development, but not posttranscriptional transgene silencing. Curr Biol 14: 346-351.

Verslues PE, Guo Y, Dong CH, Ma W, Zhu JK (2006) Mutation of SAD2, an importin $\beta$-domain protein in Arabidopsis, alters abscisic acid sensitivity. Plant J 47: 776-787.

Wu F, Yu L, Cao W, Mao Y, Liu Z, He Y (2007) The Nterminal double-strands RNA binding domains of Arabidopsis HYPONASTIC LEAVES1 are sufficient for premicroRNA processing. Plant Cell 19: 914-925.

Xiong L, Schumaker KS, Zhu JK (2002) Cell signaling during cold, drought, and salt stress. Plant Cell 14 (Suppl): S165-S183.

Xiong L, Gong Z., Rock CD, Subramanian S, Guo Y, Xu W, Galbraith D, Zhu JK (2001) Modulation of abscisic acid signal transduction and biosynthesis by an Smlike protein in Arabidopsis. Dev Cell 1: 771-781.

Yang L, Liu Z, Lu F, Dong A, Huang H (2006) SERRATE is a novel nuclear regulator in primary microRNA processing in Arabidopsis. Plant J 47: 841-850. 